\title{
Cerebral Folate Deficiency Syndrome
}

TO THE EDITOR: Ramaekers et al. (May 12 issue) ${ }^{1}$ report 28 patients with the cerebral folate deficiency syndrome characterized by decreased levels of 5-methyltetrahydrofolate (5MTHF) in the cerebrospinal fluid caused by autoantibodies' blocking of folate transport into the brain. The patients benefited from folinic acid supplementation.

The clinical features of this syndrome fit with other neurodegenerative disorders. According to previous papers by the authors, the latter disorders can be associated with cerebral folate deficiency as a secondary phenomenon..$^{2-4}$ Patients with secondary cerebral folate deficiency (e.g., the Rett syndrome) potentially benefit from folinic acid supplementation. ${ }^{2-4}$ The authors do not report the presence or absence of folate-receptor autoantibodies in secondary cerebral folate deficiency. Before claiming that the cerebral folate deficiency syndrome is a separate entity, the investigators should demonstrate that it is a unique, autoantibody-mediated disease, different from secondary cerebral folate deficiency.

Michèl A.A.P. Willemsen, M.D., Ph.D.

Ron A. Wevers, Ph.D.

Marcel M. Vebeek, Ph.D.

Radboud University Nijmegen Medical Center NL-6500 HB Nijmegen, the Netherlands

m.willemsen@cukz.umcn.nl

1. Ramaekers VT, Rothenberg SP, Sequeira JM, et al. Autoantibodies to folate receptors in the cerebral folate deficiency syndrome. N Engl J Med 2005;352:1985-91.

2. Ramaekers VT, Hansen SI, Holm J, et al. Reduced folate trans port to the CNS in female Rett patients. Neurology 2003;61:506-15.

3. Blau N, Bonafe L, Krageloh-Mann I, et al. Cerebrospinal fluid pterins and folates in Aicardi-Goutieres syndrome: a new phenotype. Neurology 2003;61:642-7.

4. Ramaekers VT, Blau N. Cerebral folate deficiency. Dev Med Child Neurol 2004;46:843-51.

THE AUTHORS REPLY: Within the group of neuropsychiatric conditions associated with cerebral folate deficiency, infantile-onset cerebral folate deficiency syndrome can be delineated as a separate and recognizable clinical entity, which can begin manifesting itself at the age of four months, when the only identifiable abnormality is a low level of 5 MTHF in the cerebrospinal fluid.1,2 This means that other conditions that involve secondary cerebral folate deficiency must be excluded before a firm diagnosis of infantile cerebral folate deficiency syndrome is established. ${ }^{2}$

The identification of blocking folate-receptor autoantibodies in the serum of most patients with infantile-onset cerebral folate deficiency syndrome merely reflects a specific autoimmune mechanism that explains the blocked folate transfer to the brain. Among the few patients without these folate-receptor autoantibodies, alternative causes may be responsible for the low levels of 5MTHF in cerebrospinal fluid.

Since blocking folate-receptor autoantibodies impair folate transport across the placenta and blood-cerebrospinal fluid barriers, ${ }^{3}$ we cannot exclude the possibility that they also play a role in the pathogenesis of secondary cerebral folate deficiency. 4,5

Vincent T. Ramaekers, M.D.

University Hospital Aachen

D-52074 Aachen, Germany

vramaekers@ukaachen.de

Nenad Blau, Ph.D.

University Children's Hospital

$\mathrm{CH}-8032$ Zurich, Switzerland

1. Ramaekers V, Hausler M, Opladen T, Heimann G, Blau N. Psychomotor retardation, spastic paraplegia, cerebellar ataxia and dyskinesia associated with low 5-methyltetrahydrofolate in cerebrospinal fluid: a novel neurometabolic condition responding to folinic acid substitution. Neuropediatrics 2002;33:301-8.

2. Ramaekers VT, Blau N. Cerebral folate deficiency. Dev Med Child Neurol 2004;46:843-51.

3. Rothenberg SP, da Costa MP, Sequeira JM, et al. Autoantibodies against folate receptors in women with a pregnancy complicated by a neural-tube defect. N Engl J Med 2004;350:134-42.

4. Ramaekers VT, Hansen SI, Holm J, et al. Reduced folate transport to the CNS in female Rett patients. Neurology 2003;61:506-15. 5. Blau N, Bonafe L, Krageloh-Mann I, et al. Cerebrospinal fluid pterins and folates in Aicardi-Goutieres syndrome: a new phenotype. Neurology 2003;61:642-7.

\section{Response of a Nonmalignant Pleural Effusion to Bevacizumab}

TO THE EDITOR: The potential role of vascular endothelial growth factor in malignant as well as nonmalignant pleural effusion ${ }^{1,2}$ prompted us to use bevacizumab, a monoclonal antibody against vas- cular endothelial growth factor, in a 68-year-old man with primary cardiac amyloidosis who had severe dyspnea and underwent repeated thoracenteses for pleural effusions. Cytologic assessments of the 